\title{
The QCD pseudocritical line from imaginary chemical potentials
}

\author{
Claudio Bonati \\ Dipartimento di Fisica dell'Università di Pisa and INFN - Sezione di Pisa, I-56127 Pisa, Italy \\ E-mail: bonatiedf.unipi.it
}

\section{Massimo D'Elia*}

Dipartimento di Fisica dell'Università di Pisa and INFN - Sezione di Pisa, I-56127 Pisa, Italy

E-mail: delia@df.unipi.it

\section{Marco Mariti}

Dipartimento di Fisica dell'Università di Pisa and INFN - Sezione di Pisa, I-56127 Pisa, Italy

E-mail: maritiedf.unipi.it

\section{Michele Mesiti}

Dipartimento di Fisica dell'Università di Pisa and INFN - Sezione di Pisa, I-56127 Pisa, Italy

E-mail: mesiti@pi.infn.it

\section{Francesco Negro}

Dipartimento di Fisica dell'Università di Pisa and INFN - Sezione di Pisa, I-56127 Pisa, Italy

E-mail: fnegro@pi.infn.it

\section{Francesco Sanfilippo}

School of Physics and Astronomy, University of Southampton, SO17 1BJ Southampton, United Kindgdom

E-mail: f.sanfilippo@soton.ac.uk

\begin{abstract}
We determine the curvature of the chiral pseudocritical line of strong interactions by analytic continuation from imaginary chemical potentials. We consider $N_{f}=2+1$ QCD discretized with stout improved staggered fermions, the tree level Symanzik gauge action and physical quark masses. We explore two different lattice spacings corresponding to temporal extensions $N_{t}=6$ and $N_{t}=8$, obtaining a preliminary continuum extrapolation for the curvature $\kappa=0.013(2)(1)$. Our results also suggests that the inclusion of a non-zero strange quark chemical potential does not lead to significant correction on $\kappa$.
\end{abstract}

9th International Workshop on Critical Point and Onset of Deconfinement - CPOD2014,

17-21 November 2014

ZiF (Center of Interdisciplinary Research), University of Bielefeld, Germany

\footnotetext{
* Speaker.
} 


\section{Introduction}

Chiral symmetry breaking is a well established property of the ground state of Quantum Chromodynamics (QCD). Lattice QCD simulations also predict the presence of a finite temperature transition to a new phase of matter where chiral symmetry is restored. For physical quark masses, which break chiral symmetry explicitly, a smooth crossover takes place [1-5] at a pseudocritical temperature $T_{c} \sim 155 \mathrm{MeV}$. Many other properties of strongly interacting matter change around that temperature, indicating the onset of deconfinement.

The dependence of $T_{C}$ on external parameters is a question of utmost importance. One of the most relevant parameters is the baryon chemical potential $\mu_{B}$, which enters the phenomenological description of heavy ion collisions. The analysis of the collision yields within a thermalstatistical hadronization model leads to the determination of a chemical freeze-out curve in the $T-\mu_{B}$ plane [6-13], corresponding to the point of last chemical equilibrium of hadron particles following re-hadronization. Even if this point is not expected to coincide with the one at which deconfinement and chiral symmetry restoration take place, a comparison with $T_{c}\left(\mu_{B}\right)$ is very interesting, also in view of the possible critical endpoint which could be present along the line and at which the crossover would turn into a real transition.

Exploration of the $T-\mu_{B}$ plane by lattice QCD simulations is presently hindered by the sign problem. Various methods exist to partially circumvent it, which permit to obtain reliable results in the regime of small $\mu_{B} / T$, for example the reweighting method, the Taylor expansion method, the canonical approach and the method of analytic continuation from an imaginary chemical potential. In the regime of small $\mu_{B} / T$ the pseudocritical line can be parametrized as follows

$$
\frac{T_{c}\left(\mu_{B}\right)}{T_{c}}=1-\kappa\left(\frac{\mu_{B}}{T_{c}}\right)^{2}+O\left(\mu_{B}^{4}\right)
$$

where $\kappa$ defines the curvature of the pseudo-critical line $T_{c}\left(\mu_{B}\right)$. In the Taylor expansion approach $\kappa$ is determined by a proper combination of expectation values computed at $\mu_{B}=0$ [14-18], while in the imaginary chemical potential approach one determines $T_{c}$ for imaginary values of $\mu_{B}$, then fitting results according to a particular ansatz for $T_{c}\left(\mu_{B}\right)$, e.g., the analytic continuation of Eq. (1.1) [18-28].

In this study we present results regarding an improved determination of the curvature by means of analytic continuation. We consider a discretization of $N_{f}=2+1$ QCD which adopts stout improved staggered fermions (with rooting), the tree level Symanzik gauge action and physical quark masses. We are interested in the properties of the chiral transition, therefore, in order to locate $T_{c}$, we look both at the renormalized chiral condensate and at the renormalized chiral susceptibility. We explore two different lattice spacings, corresponding to temporal extensions $N_{t}=6$ and $N_{t}=8$, and several spatial volumes, in order to keep both finite size and finite lattice spacing effects under control.

Regarding the setup of chemical potentials, we consider both the case $\mu_{s}=0$ and $\mu_{s} \neq 0$, where $\mu_{s}$ is the chemical potential coupled to the number of strange quarks. This is interesting in order to compare with results from heavy ion collision, where the initial conditions impose strangeness neutrality. In the free quark limit (hence for asymptotically high $T$ ) that implies $\mu_{s}=0$, which is the setup adopted by most of previous studies. However interactions lead to corrections and, 

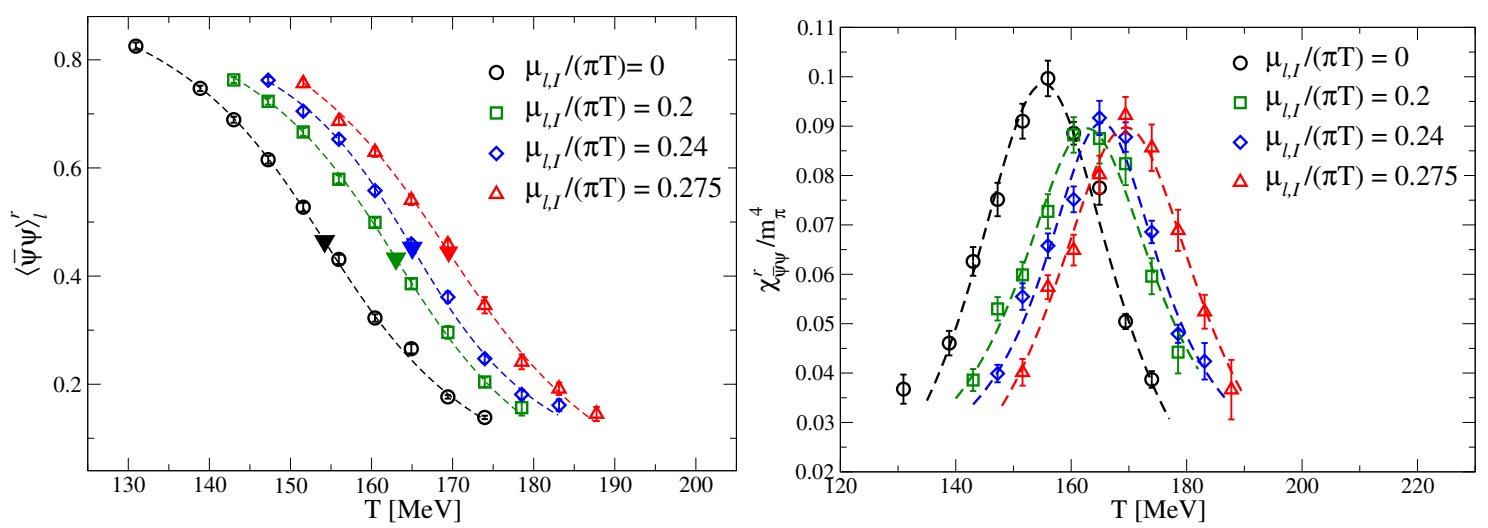

Figure 1: Renormalized chiral condensate (left) and renormalized chiral susceptibility (right) for various values of $T$ and $\mu_{l}$ on a $32^{3} \times 8$ lattice with $\mu_{s}=0$. Lines correspond to the best fits described in the text.

around $T_{c}$, strangeness neutrality is guaranteed by $\mu_{s} \sim 0.25 \mu_{l}$ [29]. Exploring both cases $\left(\mu_{s}=0\right.$ and $\mu_{s}=\mu_{l}$ ) permits to state the systematic corrections due to the choice of $\mu_{s}$. A full account of our results has been presented in Ref. [30].

\section{Numerical Setup}

We perform lattice simulations of $N_{f}=2+1$ QCD with purely imaginary quark chemical potentials, $\mu_{f}=i \mu_{f, I}, \mu_{f, I} \in \mathbb{R}$, with $f=u, d$,s. The euclidean partition function is the following:

$$
\begin{aligned}
\mathscr{Z} & =\int \mathscr{D} U e^{-\mathscr{S}_{Y M}} \prod_{f=u, d, s} \operatorname{det}\left(M_{\mathrm{st}}^{f}\left[U, \mu_{f, I}\right]\right)^{1 / 4}, \\
\left(M_{\mathrm{St}}^{f}\right)_{i, j} & =a m_{f} \delta_{i, j}+\sum_{v=1}^{4} \frac{\eta_{i ; \nu}}{2}\left[e^{i a \mu_{f, I} \delta_{v, 4}} U_{i ; \nu}^{(2)} \delta_{i, j-\hat{v}}-e^{-i a \mu_{f, I} \delta_{v, 4}} U_{i-\hat{v} ; v}^{(2) \dagger} \delta_{i, j+\hat{v}}\right]
\end{aligned}
$$

where $\mathscr{S}_{Y M}$ is the tree level improved Symanzik action [31,32] and the staggered Dirac operator $\left(M_{\mathrm{St}}^{f}\right)_{i, j}$ is built up in terms of the two times stout-smeared [33] links $U_{i ; v}^{(2)}$ with isotropic smearing parameter $\rho=0.15$. The residual fourth degeneracy of the lattice Dirac operator is removed, as usual, by means of the rooting procedure. The imaginary chemical potential is introduced, for each quark flavor, by multiplying all temporal links in the corresponding Dirac operator by $e^{i a \mu_{f, I}}$, $f=u, d, s$. That can be viewed as a rotation by an angle $\theta_{f} \equiv a N_{t} \mu_{f, I}=\mu_{f, I} / T$ of the temporal boundary conditions for the quark flavor $f$.

We explore the region around the transition temperature on lattices with two different temporal extensions, $N_{t}=6$ and $N_{t}=8$. Various spatial sizes are explored in some cases, to check for finite size effects. For each lattice we study 3-4 different values of the imaginary chemical potentials with two different setups, either $\mu_{u}=\mu_{d}=\mu_{l}$ and $\mu_{s}=0$, or $\mu_{u}=\mu_{d}=\mu_{s}=\mu_{l}$. The temperature $T=$ $1 /\left(a N_{t}\right)$ of the system is changed by varying the value of the bare coupling constant $\beta$, rescaling the bare quark masses $m_{s}$ and $m_{u}=m_{d}=m_{l}$ so as to move on a line of constant physics, with $m_{\pi} \simeq 135 \mathrm{MeV}$ and $m_{s} / m_{l}=28.15$; this is done by a spline interpolation of the values reported in Refs. [34,35]. 

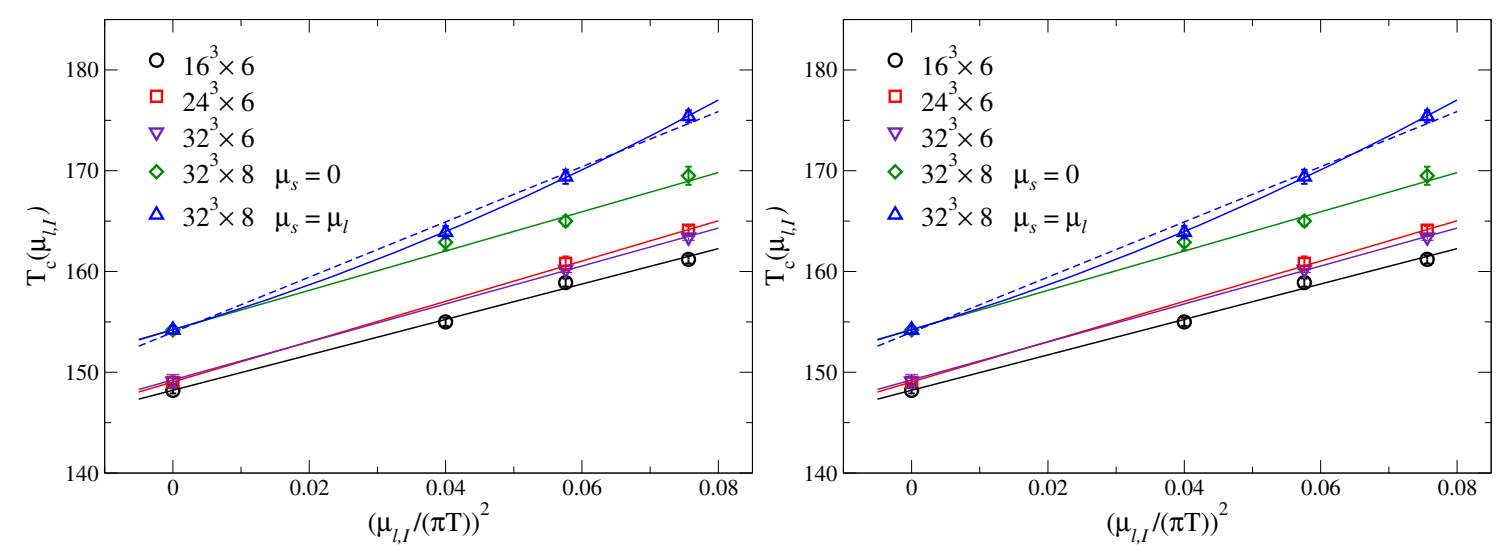

Figure 2: Determinations of $T_{c}$ obtained from the renormalized chiral condensate (left) and from the renormalized chiral susceptibility (right) for various values of the chemical potential and of the lattice size. The lines correspond to quadratic and quartic fits in $\mu_{l, I}$, as discussed in the text.

The location of the transition is defined in terms of the light quark condensate:

$$
\langle\bar{\psi} \psi\rangle_{l}=\frac{T}{V} \frac{\partial \log Z}{\partial m_{l}}=\langle\bar{u} u\rangle+\langle\bar{d} d\rangle,
$$

which is renormalized by adopting the prescription introduced in Ref. [36]:

$$
\langle\bar{\psi} \psi\rangle_{l}^{r}(T) \equiv \frac{\left[\langle\bar{\psi} \psi\rangle_{l}-\frac{2 m_{l}}{m_{s}}\langle\bar{s} s\rangle\right](T)}{\left[\langle\bar{\psi} \psi\rangle_{l}-\frac{2 m_{l}}{m_{s}}\langle\bar{s} s\rangle\right](T=0)},
$$

where $m_{s}$ is the bare strange quark mass. The light quark chiral susceptibility is given instead by

$$
\begin{aligned}
\chi_{\bar{\psi} \psi} & =\frac{\partial\langle\bar{\psi} \psi\rangle_{l}}{\partial m_{l}}=\chi_{\bar{\psi} \psi}^{\text {disc }}+\chi_{\bar{\psi} \psi}^{c o n n} \\
\chi_{\bar{\psi} \psi}^{\text {disc }} & \equiv \frac{T}{V}\left(\frac{N_{l}}{4}\right)^{2}\left[\left\langle\left(\operatorname{Tr} M_{l}^{-1}\right)^{2}\right\rangle-\left\langle\operatorname{Tr} M_{l}^{-1}\right\rangle^{2}\right] \\
\chi_{\bar{\psi} \psi}^{c o n n} & \equiv-\frac{T}{V} \frac{N_{l}}{4}\left\langle\operatorname{Tr} M_{l}^{-2}\right\rangle .
\end{aligned}
$$

where $M_{l}$ is the Dirac operator corresponding to a single light flavor and $N_{l}=2$. Traces are computed by noisy estimators, with 8 random vectors for each flavor. The renormalization of the chiral susceptibility is performed by subtracting the $T=0$ contribution, then multiplying the result by $m_{l}^{2}$ in order to fix the multiplicative ultraviolet (UV) divergence [34]:

$$
\chi_{\bar{\psi} \psi}^{r}=m_{l}^{2}\left[\chi_{\bar{\psi} \psi}(T)-\chi_{\bar{\psi} \psi}(T=0)\right] .
$$

All the $T=0$ quantities have been measured on symmetric lattices.

The Rational Hybrid Monte-Carlo algorithm [37-39] has been used for sampling gauge configurations according to Eq. (2.1), each single run consisting of 2-5 K trajectories of unit length in molecular dynamics time, with higher statistics around the transition. 

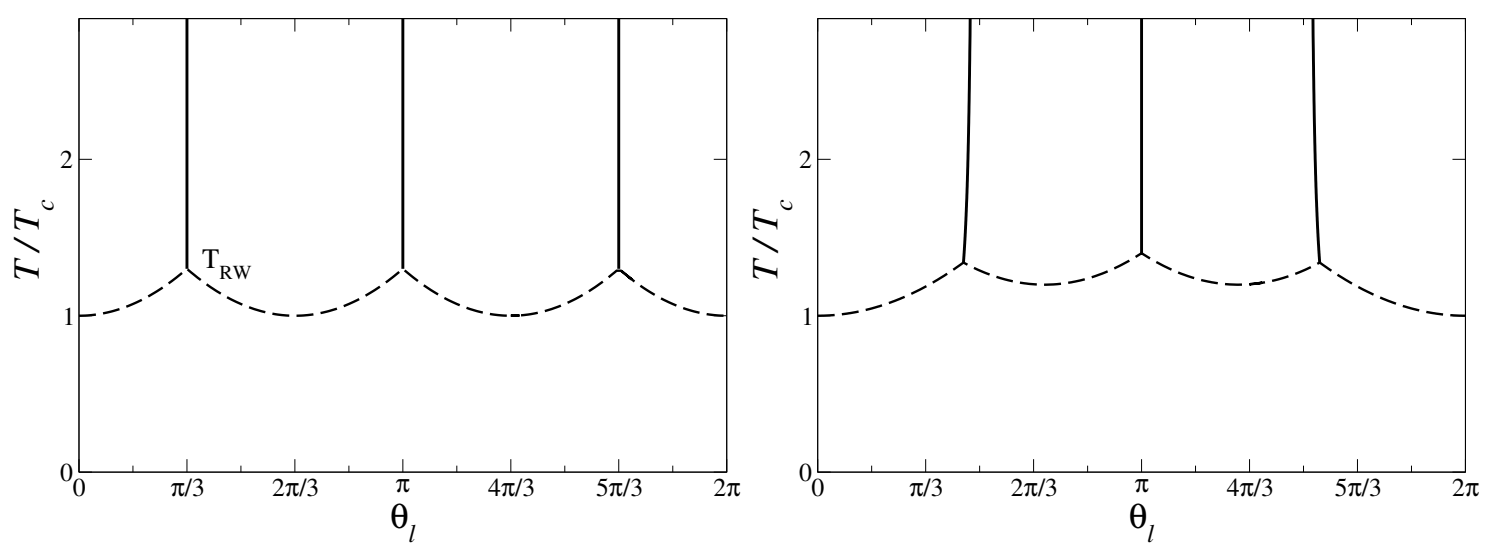

Figure 3: Sketched phase diagram in the $T-\theta_{l}$ plane for $\mu_{s}=\mu_{l}$ (left) and for $\mu_{s}=0$ (right). Solid lines indicate the RW (or RW-like) lines, while the dashed lines corresponds to the analytic continuation of the pseudocritical line.

\section{Numerical Results}

In Fig. 1 we plot the results obtained for the renormalized chiral condensate and for the renormalized chiral susceptibility on the $32^{3} \times 8$ lattice and for the $\mu_{s}=0$ setup; analogous results have been obtained for the other cases explored. In order to determine the location of the pseudocritical temperature $T_{c}$, we have looked either for the inflection point of the renormalized chiral condensate or for the maximum of the renormalized chiral susceptibility. Both these definitions are faithful, meaning that they provide the correct answer in case a real phase transition is present.

In order to determine the inflection point of the renormalized chiral condensate, we have performed a best fit to the data by using the expression

$$
\langle\bar{\psi} \psi\rangle_{l}^{r}(T)=A_{1}+B_{1} \arctan \left(C_{1}\left(T-T_{c}\right)\right) .
$$

Instead, in the case of the renormalized susceptibility, the peak has been located by fitting data to a Lorentzian function

$$
\chi_{\bar{\psi} \psi}^{r}=\frac{A_{2}}{B_{2}^{2}+\left(T-T_{c}\right)^{2}} .
$$

In both cases such functions are found to well describe the behavior of data points in the whole range of explored temperatures. In Fig. 1 the best fits are plotted together with the data points, the position of the inflection point being denoted, for each data set, by a filled triangle. The errors on the fit parameters have been estimated by means of a bootstrap analysis; results for $T_{c}$ are stable, within errors, if different fitting functions are adopted (e.g., a hyperbolic tangent for the condensate or a quadratic function for the susceptibility).

The full set of determinations of $T_{c}\left(\mu_{l, I}, \mu_{s, I}\right)$ is reported in Fig. 2. In order to extract the curvature, we performed a fit to the values obtained for $T_{c}\left(\mu_{l, I}\right)$, separately for each lattice size and setup of chemical potentials, according to the function

$$
T_{c}\left(\mu_{l, I}\right)=T_{c}(0)\left(1+9 \kappa\left(\frac{\mu_{l, I}}{T_{c}\left(\mu_{l, I}\right)}\right)^{2}+81 b\left(\frac{\mu_{l, I}}{T_{c}\left(\mu_{l, I}\right)}\right)^{4}+O\left(\mu_{l, I}^{6}\right)\right) .
$$




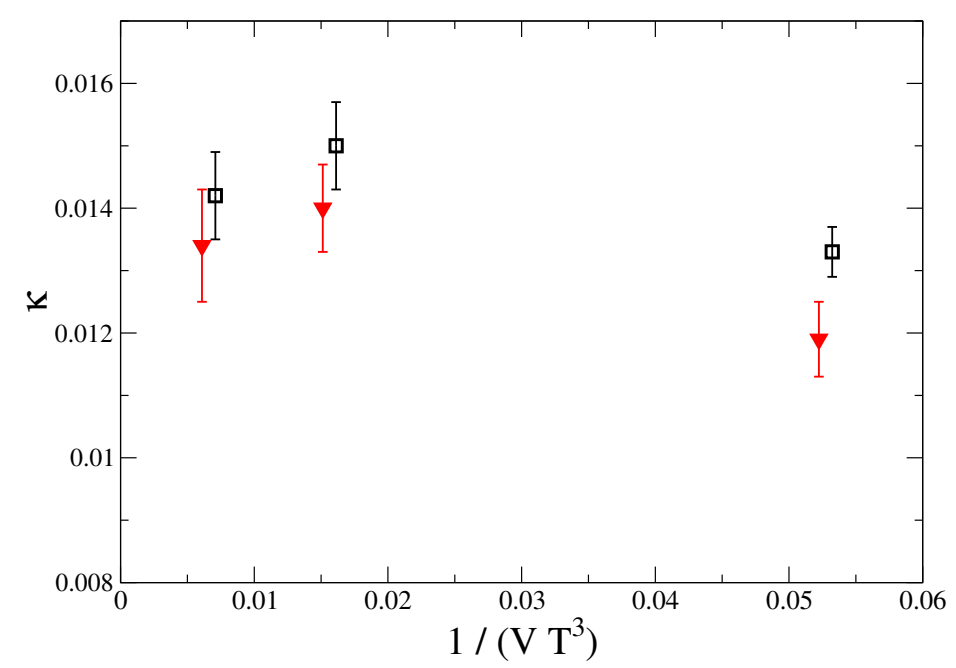

Figure 4: Fitted values of the curvature $\kappa$ from $N_{t}=6$ lattices $\left(\mu_{s}=0\right)$ as a function of the inverse spatial volume. Squares correspond to the determinations obtained by using the chiral condensate, triangles to the chiral susceptibility estimates.

In this way we have obtained estimates of $\kappa$ for all the lattices and the chemical potential setups adopted. In most cases, a simple linear fit (i.e. setting $b=0$ ) works quite well. Just for the $\mu_{s}=\mu_{l}$ setup (studied only on the $32^{3} \times 8$ lattice) the introduction of a quartic correction is necessary in order to obtain reasonable values of the $\tilde{\chi}^{2}$ test. However, as one can appreciate from the figure, the value of the curvature, i.e. the slope at $\mu_{l}=0$, does not change in a significant way when changing setup, showing that $\kappa$ is not very sensitive to the value of $\mu_{s}$.

It is tempting to associate the enhancement of non-linear corrections in the $\mu_{s}=\mu_{l}$ setup to the fact that, in this case, the phase structure in the $T-\theta_{l}$ plane, where $\theta_{l}=\operatorname{Im}\left(\mu_{l}\right) / T$, is different. For $\mu_{s}=\mu_{l}$ one has the usual phase diagram with Roberge-Weiss (RW) transitions [40] taking place for $\mu_{l}=\pi / 3$ or odds multiple of it, connected to the analytic continuation of the pseudocritical line through their endpoints. For $\mu_{s}=0$, instead, the strange quark determinant has still standard temporal boundary conditions (i.e. non-rotated), and that tends to keep the system in the real center sector, so that the first sudden jump to non-real center sectors is delayed and the region around $\theta_{l}=0$, which is the one available for analytic continuation, is a bit larger. This situation is depicted in Fig. 3. The proximity of the RW line for $\mu_{s}=\mu_{l}$ could explain the larger non-linear contribution to the pseudocritical line in this case.

In Fig. 4 we compare data at fixed UV cutoff $\left(N_{t}=6\right)$ and different spatial volumes $\left(L_{s}=\right.$ $16,24,32)$, in order to show that finite volume effects are well under control. In Fig. 5, instead, we report the values of $\kappa$ obtained for $\mu_{s}=0$ and at fixed physical spatial volume for two different values of the UV cutoff (corresponding to $N_{t}=6$ and $N_{t}=8$ ). Results show that UV effects are under control.

A preliminary continuum extrapolation, performed assuming order $a^{2}$ corrections, yields $\kappa=$ 0.0132 (18) (from the renormalized chiral condensate) and $\kappa=0.0126(22)$ (from the renormalized chiral susceptibility), however this cannot be considered yet as a rigorous continuum extrapolation, since we have not enough data points to perform a best fit. Different kinds of extrapolation (e.g., 


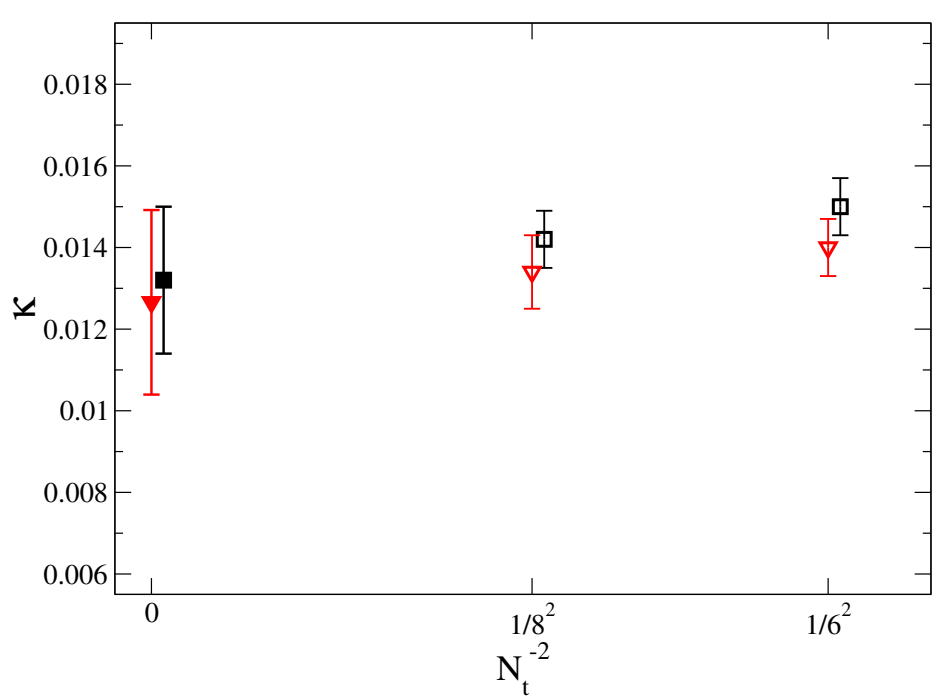

Figure 5: Determinations of $\kappa$ for $\mu_{s}=0$ and on lattices with aspect ratio 4 as a function of $N_{t}^{-2}$; the points at zero abscissa correspond to a rudimentary continuum limit extrapolation, assuming corrections linear in $N_{t}^{-2}$. Squares correspond to the determinations obtained by using the chiral condensate, triangles to the chiral susceptibility estimates.

according to a constant function) permits us to estimate systematic uncertainties, leading to a final estimate $\kappa=0.13(2)(1)$.

Finally, in Fig. 6, we compare our present results with previous ones in the literature. We do not report many early determinations and consider only a collection of recent ones, which look at the chiral transition and have been obtained by discretizations of $N_{f}=2+1 \mathrm{QCD}$ at or close to the physical point $[15,16,28]$. Our results are generally larger than results obtained by the Taylor expansion $[15,16]$ and in marginal agreeement with results obtained by analytic continuation and a different discretization [28]. A careful analysis of systematic uncertainties shows that the discrepancy with Taylor expansion determinations is presently at a $2 \sigma$ level [30]. In our case, a determination of $\kappa$ on finer lattices, with $N_{t}=10$ and $N_{t}=12$, is a necessary step, which should be undertaken in the next future in order to obtain complete control over the continuum extrapolation and reach definite continuum results.

\section{Acknowledgments}

FS received funding from the European Research Council under the European Community Seventh Framework Programme (FP7/2007-2013) ERC grant agreement No 279757. FN acknowledges financial support from the EU under project Hadron Physics 3 (Grant Agreement n. 283286). This work was partially supported by the INFN SUMA project. Simulations have been performed on the BlueGene/Q Fermi at CINECA (Project Iscra-B/EPDISIM), and on the CSN4 Zefiro cluster of the Scientific Computing Center at INFN-PISA. 


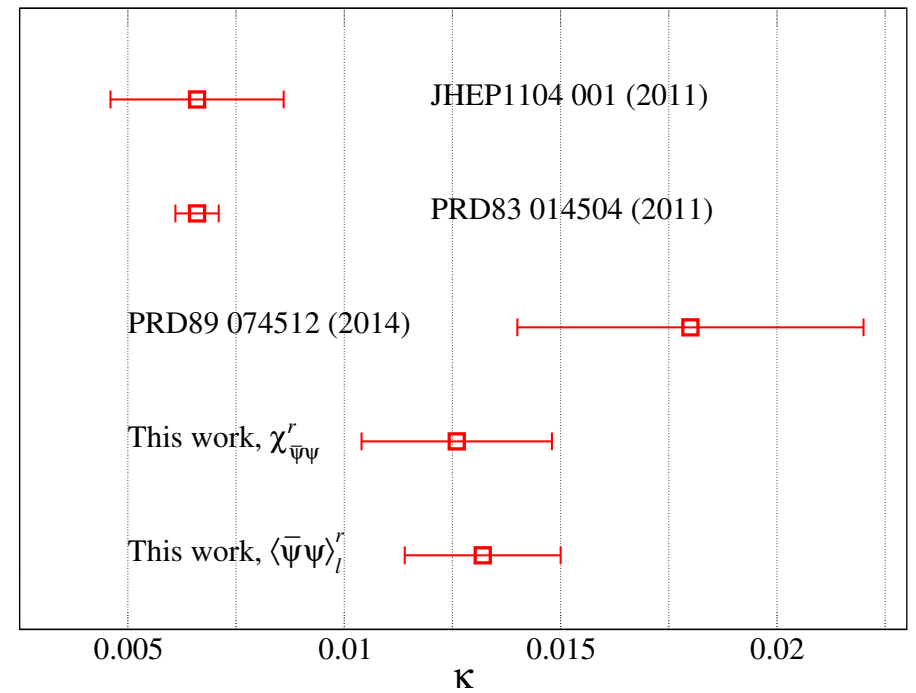

Figure 6: Curvature of the pseudo-critical line from different studies. From bottom to top: $i$ ) and $i i)$ analytic continuation, chiral condensate and chiral susceptibility, stout staggered quarks (this study [30]); iii) analytic continuation, chiral susceptibility with $\mu_{s}=\mu_{l}$, HISQ staggered quarks [28]; $i v$ ) Taylor expansion, chiral susceptibility, staggered quark p4-action [15]; $v$ ) Taylor expansion, chiral condensate, stout staggered quarks [16].

\section{References}

[1] Y. Aoki, G. Endrodi, Z. Fodor, S. D. Katz and K. K. Szabo, Nature 443 (2006) 675 [hep-lat/0611014].

[2] Y. Aoki, Z. Fodor, S. D. Katz and K. K. Szabo, Phys. Lett. B 643, 46 (2006) [hep-lat/0609068].

[3] S. Borsanyi et al., JHEP 1009, 073 (2010).

[4] A. Bazavov, T. Bhattacharya, M. Cheng, C. DeTar, H. T. Ding, S. Gottlieb, R. Gupta and P. Hegde et al., Phys. Rev. D 85, 054503 (2012) [arXiv:1111.1710 [hep-lat]].

[5] T. Bhattacharya, M. I. Buchoff, N. H. Christ, H. -T. Ding, R. Gupta, C. Jung, F. Karsch and Z. Lin et al., arXiv:1402.5175 [hep-lat].

[6] P. Braun-Munzinger, J. Stachel, J. P. Wessels and N. Xu, Phys. Lett. B 344, 43 (1995) [nucl-th/9410026]; Phys. Lett. B 365, 1 (1996) [nucl-th/9508020].

[7] F. Becattini, Z. Phys. C 69, 485 (1996).

[8] F. Becattini and U. W. Heinz, Z. Phys. C 76, 269 (1997) [Erratum-ibid. C 76, 578 (1997)] [hep-ph/9702274].

[9] F. Becattini, J. Cleymans, A. Keranen, E. Suhonen and K. Redlich, Phys. Rev. C 64, 024901 (2001) [hep-ph/0002267].

[10] P. Braun-Munzinger, D. Magestro, K. Redlich and J. Stachel, Phys. Lett. B 518, 41 (2001) [hep-ph/0105229].

[11] A. Andronic, P. Braun-Munzinger and J. Stachel, Nucl. Phys. A 772, 167 (2006) [nucl-th/0511071].

[12] J. Cleymans, H. Oeschler, K. Redlich and S. Wheaton, Phys. Rev. C 73, 034905 (2006) [hep-ph/0511094]. 
[13] F. Becattini, M. Bleicher, T. Kollegger, T. Schuster, J. Steinheimer and R. Stock, Phys. Rev. Lett. 111, 082302 (2013) [arXiv:1212.2431 [nucl-th]].

[14] C. R. Allton, S. Ejiri, S. J. Hands, O. Kaczmarek, F. Karsch, E. Laermann, C. Schmidt and L. Scorzato, Phys. Rev. D 66, 074507 (2002) [hep-lat/0204010].

[15] O. Kaczmarek, F. Karsch, E. Laermann, C. Miao, S. Mukherjee, P. Petreczky, C. Schmidt, W. Soeldner and W. Unger, Phys. Rev. D 83, 014504 (2011) [arXiv:1011.3130 [hep-lat]].

[16] G. Endrodi, Z. Fodor, S. D. Katz and K. K. Szabo, JHEP 1104, 001 (2011) [arXiv:1102.1356 [hep-lat]].

[17] S. Borsanyi, G. Endrodi, Z. Fodor, S. D. Katz, S. Krieg, C. Ratti and K. K. Szabo, JHEP 1208, 053 (2012) [arXiv:1204.6710 [hep-lat]].

[18] E. Laermann, F. Meyer and M. P. Lombardo, J. Phys. Conf. Ser. 432, 012016 (2013). E. Laermann, F. Meyer and M. P. Lombardo, arXiv:1304.3247 [hep-lat].

[19] P. de Forcrand and O. Philipsen, Nucl. Phys. B 642, 290 (2002) [hep-lat/0205016]; Nucl. Phys. B 673, 170 (2003) [hep-lat/0307020].

[20] M. D’Elia and M. P. Lombardo, Phys. Rev. D 67, 014505 (2003) [hep-lat/0209146]; Phys. Rev. D 70, 074509 (2004) [hep-lat/0406012].

[21] V. Azcoiti, G. Di Carlo, A. Galante and V. Laliena, Nucl. Phys. B 723, 77 (2005) [hep-lat/0503010].

[22] L. K. Wu, X. Q. Luo and H. S. Chen, Phys. Rev. D 76, 034505 (2007) [hep-lat/0611035].

[23] P. Cea, L. Cosmai, M. D’Elia and A. Papa, Phys. Rev. D 77, 051501 (2008) [arXiv:0712.3755 [hep-lat]].

[24] P. Cea, L. Cosmai, M. D’Elia, C. Manneschi and A. Papa, Phys. Rev. D 80, 034501 (2009) [arXiv:0905.1292 [hep-lat]].

[25] P. Cea, L. Cosmai, M. D’Elia and A. Papa, Phys. Rev. D 81, 094502 (2010) [arXiv:1004.0184 [hep-lat]].

[26] K. Nagata and A. Nakamura, Phys. Rev. D 83, 114507 (2011) [arXiv:1104.2142 [hep-lat]].

[27] P. Cea, L. Cosmai, M. D’Elia, A. Papa and F. Sanfilippo, Phys. Rev. D 85, 094512 (2012) [arXiv:1202.5700 [hep-lat]].

[28] P. Cea, L. Cosmai and A. Papa, Phys. Rev. D 89, 074512 (2014) [arXiv:1403.0821 [hep-lat]].

[29] A. Bazavov, H.-T. Ding, P. Hegde, O. Kaczmarek, F. Karsch, E. Laermann, Y. Maezawa and S. Mukherjee et al., Phys. Rev. Lett. 113, 072001 (2014) [arXiv:1404.6511 [hep-lat]].

[30] C. Bonati, M. D’Elia, M. Mariti, M. Mesiti, F. Negro and F. Sanfilippo, Phys. Rev. D 90, no. 11, 114025 (2014) [arXiv:1410.5758 [hep-lat]].

[31] P. Weisz, Nucl. Phys. B 212, 1 (1983).

[32] G. Curci, P. Menotti and G. Paffuti, Phys. Lett. B 130, 205 (1983) [Erratum-ibid. B 135, 516 (1984)].

[33] C. Morningstar and M. J. Peardon, Phys. Rev. D 69, 054501 (2004) [hep-lat/0311018].

[34] Y. Aoki, S. Borsanyi, S. Durr, Z. Fodor, S. D. Katz, S. Krieg and K. K. Szabo, JHEP 0906, 088 (2009) [arXiv:0903.4155 [hep-lat]]. 
[35] S. Borsanyi, G. Endrodi, Z. Fodor, A. Jakovac, S. D. Katz, S. Krieg, C. Ratti and K. K. Szabo, JHEP 1011, 077 (2010) [arXiv:1007.2580 [hep-lat]]; S. Borsanyi, Z. Fodor, C. Hoelbling, S. D. Katz, S. Krieg and K. K. Szabo, Phys. Lett. B 730, 99 (2014) [arXiv:1309.5258 [hep-lat]].

[36] M. Cheng, N. H. Christ, S. Datta, J. van der Heide, C. Jung, F. Karsch, O. Kaczmarek and E. Laermann et al., Phys. Rev. D 77, 014511 (2008) [arXiv:0710.0354 [hep-lat]].

[37] M. A. Clark, A. D. Kennedy and Z. Sroczynski, Nucl. Phys. Proc. Suppl. 140, 835 (2005) [hep-lat/0409133].

[38] M. A. Clark and A. D. Kennedy, Phys. Rev. D 75, 011502 (2007) [hep-lat/0610047].

[39] M. A. Clark and A. D. Kennedy, Phys. Rev. Lett. 98, 051601 (2007) [hep-lat/0608015].

[40] A. Roberge and N. Weiss, Nucl. Phys. B 275 (1986) 734. 